\title{
Should intake of carbon monoxide be used as a guide to intake of other smoke constituents?
}

\author{
HEATHER ASHTON, R STEPNEY, J W THOMPSON
}

\begin{abstract}
The relation between blood carboxyhaemoglobin (COHb) and plasma nicotine concentrations was studied in a group of 12 smokers smoking cigarettes of three levels of standard delivery. While the intake of carbon monoxide from a single cigarette was unrelated to the intake of nicotine, presmoking "trough" concentrations of the two substances (reflecting longer-term exposure) were highly correlated. Various other measures of nicotine exposure were at best only moderately correlated with blood nicotine concentrations.

Thus trough COHb concentrations might be used to provide a reliable indication of the exposure to nicotine of individual smokers smoking the same type of cigarette, and of the relative exposure to nicotine of populations smoking cigarettes of different standard deliveries.
\end{abstract}

\section{Introduction}

Carbon monoxide intake has been used to derive an index of smoke inhalation from which relative exposure to tar and nicotine may be estimated in populations smoking different tobacco products. ${ }^{1}$ An important practical advantage is that intake of carbon monoxide is easily measured, either as the percentage of carboxyhaemoglobin $(\mathrm{COHb})$ in venous blood or (entirely non-invasively) as a concentration in end-tidal air. The concentration in end-tidal air correlates highly $(r=0.97)$ with $\mathrm{COHb}$ concentrations ${ }^{2}$ and would seem to have great potential use in large-scale epidemiological studies. Estimating exposure to nicotine (by measuring its concentration in body fluids), on the other hand, is complex, and no direct measure of exposure to tar in human subjects is currently available.

Extrapolating from carbon monoxide intake to that of tar and nicotine, however, is not without problems. Carbon monoxide is not closely allied with tar and nicotine in tobacco smoke: while carbon monoxide is a constituent of the vapour phase, tar and nicotine are in the particulate phase. Secondly, a small proportion of the nicotine available in smoke may be absorbed through the buccal and nasal mucosa (that is, in the absence of inhalation), while carbon monoxide is absorbed only when smoke is drawn into the lungs. ${ }^{3}$ Thirdly, it has been estimated that after inhalation subjects retain over $90 \%$ of the nicotine in cigarette smoke, $60-80 \%$ of the tar (depending on inhalation pattern), but only $60 \%$ of the carbon monoxide (D E Creighton. Paper presented at the conference on aerosol physics, British American Tobacco Co Ltd, Southampton, 1973). Moreover, while it is the extent of absorption of carbon monoxide and nicotine that is relevant to the effects of these substances on health, with tar it is the pattern of deposition in the respiratory tract that is important. Furthermore, a certain amount of carbon monoxide is

Clinical Psychopharmacology Unit, Department of Pharmacological Sciences, University of Newcastle upon Tyne, Newcastle upon Tyne NE2 4AB

HEATHER ASHTON, DM, FRCP, senior lecturer in psychopharmacology and consultant clinical pharmacologist

R STEPNEY, MA, MSC, junior research associate (present address: Department of Medicine, University of Cambridge Clinical School, Addenbrooke's Hospital, Cambridge CB2 2QQ)

I W THOMPSON, PHD, MRCP, professor of pharmacology and consultant clinical pharmacologist (AHA(T)) endogenously produced ${ }^{4}$ and sources of carbon monoxide other than smoking exist in the environment. Consequently a "background" concentration of carbon monoxide is found in people who are not at all exposed to nicotine and tar.

The a priori grounds for supposing that exposure to carbon monoxide will be systematically related to exposure to nicotine and tar are therefore unclear. Recently we showed that cigarette smokers titrate intakes of nicotine and carbon monoxide when switched to cigarettes with higher or lower machine yields of these substances. ${ }^{5}$ In the course of the experiment plasma nicotine and blood $\mathrm{COHb}$ concentrations were measured. We have analysed further the data in our original study to determine the extent to which nicotine and $\mathrm{COHb}$ concentrations are correlated and whether measuring exposure to carbon monoxide provides a reliable estimate of exposure to nicotine. In addition, we correlated plasma nicotine concentrations with two other measures of nicotine exposure (mouth-level nicotine intake as determined by butt analysis and urinary excretion of nicotine and cotinine) and with certain variables of smoking behaviour and physiological variables thought to be related to nicotine intake.

\section{Subjects and methods}

Twelve volunteer smokers (six men, six women) participated in an experiment in which smoking behaviour and the intake of smoke constituents were studied when the subjects smoked their usual middle-tar, middle-nicotine cigarettes and when they switched to each of two experimental brands, one giving lower and one higher standard yields of nicotine, tar, and carbon monoxide. Subjects smoked each type of cigarette over at least two weeks, during which they attended the laboratory on two occasions. On each occasion the pattern of puffing used to smoke a single cigarette was recorded and a venous blood sample taken before and immediately after smoking. The purpose of the presmoking blood sample was to provide baseline values from which to measure the increase in exposure to smoke constituents during the consumption of a single cigarette. Since subjects were requested to refrain from smoking in the half hour before their laboratory attendance, however, the presmoking concentrations served also as resting or trough values, reflecting the exposure to smoke constituents over a fairly long period of smoking outside the laboratory. Table I shows the mean time between the last cigarette and the subjects' attendance at the laboratory and the number of cigarettes smoked before attendance.

TABLE I-Mean $( \pm S D)$ time between smoking of last cigarette and attendance at laboratory and number of cigarettes smoked before attendance

\begin{tabular}{|c|c|c|c|}
\hline & $\begin{array}{c}\text { Usual } \\
\text { brands }\end{array}$ & $\begin{array}{c}\text { High } \\
\text { delivery }\end{array}$ & $\begin{array}{l}\text { Low } \\
\text { delivery }\end{array}$ \\
\hline $\begin{array}{l}\text { Time since last cigarette }(\min )(n=11) \\
\text { No of cigarettes smoked }(n=12)\end{array}$ & $\begin{array}{l}49 \pm 36^{*} \\
5 \cdot 2 \pm 2 \cdot 5\end{array}$ & $\begin{array}{c}45 \pm 15 \\
4 \cdot 9 \pm 2 \cdot 6\end{array}$ & $\begin{array}{c}37 \pm 13 \\
5 \cdot 5 \pm 1 \cdot 8\end{array}$ \\
\hline
\end{tabular}

*This value is inflated because one subject had not smoked for four hours before attending the laboratory.

Plasma nicotine concentrations were assayed by gas chromatography and blood $\mathrm{COHb}$ with an IL $182 \mathrm{CO}$-oximeter. A more complete account of the subjects' characteristics, the standard deliveries of the cigarettes used, and the variables measured has already been published. ${ }^{5}$ Table II shows the standard deliveries of the three types of cigarette smoked.

The relation between plasma nicotine and $\mathrm{COHb}$ concentrations (and between plasma nicotine concentrations and other variables) was established by between-subject Pearson product moment correlation. Each variable was measured twice in all subjects for each type of cigarette, so the means of the two values were used in the correlations. 
TABLE II-Standard deliveries (machine yield) of the three types of cigarette

\begin{tabular}{lccc}
\hline & $\begin{array}{c}\text { Usual brands } \\
\text { (mean) }\end{array}$ & $\begin{array}{c}\text { High } \\
\text { delivery }\end{array}$ & $\begin{array}{c}\text { Low } \\
\text { delivery }\end{array}$ \\
\hline Nicotine (mg) & 1.4 & 1.84 & 0.6 \\
Carbon monoxide (mg) & 17.5 & 24.0 & 9.9 \\
Tar (mg) & 18.0 & 26.5 & 6.5
\end{tabular}

\section{Results}

Table III shows the correlations between plasma nicotine and blood $\mathrm{COHb}$ concentrations before and after smoking and between the rises in concentration with smoking for the three brands of cigarettes. For all three types of cigarette the correlation between presmoking plasma nicotine and $\mathrm{COHb}$ concentrations equalled or exceeded 0.89 $(p<0.001)$. Postsmoking concentrations, however, were less highly correlated $(r \geqslant 0.64, p<0.05)$, and no systematic relation existed between the rise in $\mathrm{COHb}$ and plasma nicotine concentrations with smoking.

TABLE III-Correlations between plasma nicotine and blood COHb concentrations in 12 subjects smoking middle-, high-, and low-delivery cigarettes

\begin{tabular}{lccc}
\hline Cigarette & Presmoking & Postsmoking & Rise with smoking \\
\hline Uusual brands (middle delivery) & $0.89^{* * *}$ & $0.64^{*}$ & -0.01 \\
High delivery & $0.96^{* * *}$ & $0.75^{* *}$ & -0.36 \\
Low delivery & $0.93^{* * *}$ & $0.70^{* *}$ & 0.34 \\
\hline
\end{tabular}

${ }^{*} \mathrm{p}<0.05 ;{ }^{* *} \mathrm{p}<0.01 ;{ }^{* * *} \mathrm{p}<0.001(\mathrm{df}=10)$

Figure 1 shows the relation between presmoking $\mathrm{COHb}$ and plasma nicotine concentrations in greater detail. Although the degree of correlation was closely similar for each brand, the slope of the regression line was rather less steep for the low-delivery cigarette than for the two other brands. The differences in slope were not significant but accorded with the between-brand differences in the ratio of standard carbon monoxide to nicotine deliveries. Thus the usual and highdelivery brands (with similar ratios of carbon monoxide to nicotine deliveries on machine smoking (12.5 and 13.0, respectively)) had almost identical slopes. The low-delivery brand, in contrast, had machine-smoked deliveries in the ratio $16 \cdot 5: 1$, indicating that proportionately more carbon monoxide was delivered per unit of nicotine. This corresponds with the shallower slope of the regression line, indicating that the intake of carbon monoxide per unit of nicotine was also greater with human smoking.

Measures of carbon monoxide and nicotine intake may also be used to obtain an index of the intensity with which a particular type of cigarette is smoked relative to some arbitrarily chosen baseline. The nicotine and $\mathrm{COHb}$ concentrations observed in our subjects were therefore related to the concentrations that would have been obtained if smokers had smoked the high- and low-delivery brands in the same way that they smoked their usual brands. Table IV shows the indices of smoking intensity based on both plasma nicotine concentrations and $\mathrm{COHb}$. Values of less than 1.00 indicate that the brand was smoked less intensively than subjects' usual cigarettes; values above 1.00 indicate relative oversmoking.

The indices were calculated in the following way, taking presmoking plasma nicotine concentrations as an example. The mean concentration for the group as a whole was divided by the mean standard nicotine delivery of subjects' own brands $(1.4 \mathrm{mg})$ to give an estimate of the

TABLE IV-Index of smoking intensity based on nicotine and COHb concentrations

\begin{tabular}{llc}
\hline & \multicolumn{2}{c}{ Brand delivery } \\
\cline { 2 - 3 } Index based on: & High & Low \\
\hline Presmoking concentrations: & 0.82 & 1.98 \\
Plasma nicotine & 0.77 & 1.71 \\
COHb* & 0.81 & 1.68 \\
Rise in concentrations with smoking: & 0.82 & 1.42 \\
Plasma nicotine & &
\end{tabular}

*In calculating the index of smoking intensity based on presmoking COHb concen"In calculating the index of smoking intensity based on presmoking COHb concentration a "background" value of $1 \%$ (representing the concen
smokers) was subtracted from the observed concentrations. nicotine exposure per $1 \mathrm{mg}$ of standard delivery. This figure multiplied by 1.84 or 0.6 (the standard nicotine yield in $\mathrm{mg}$ of the high- and low-delivery brands respectively) gives the mean blood concentration that would have been obtained if subjects had smoked the experimental brand exactly as intensively as they smoked their usual brands. The mean of the concentrations actually observed was then divided by this "expected" value to give a measure of the average intensity with which the experimental brand was smoked relative to the usual
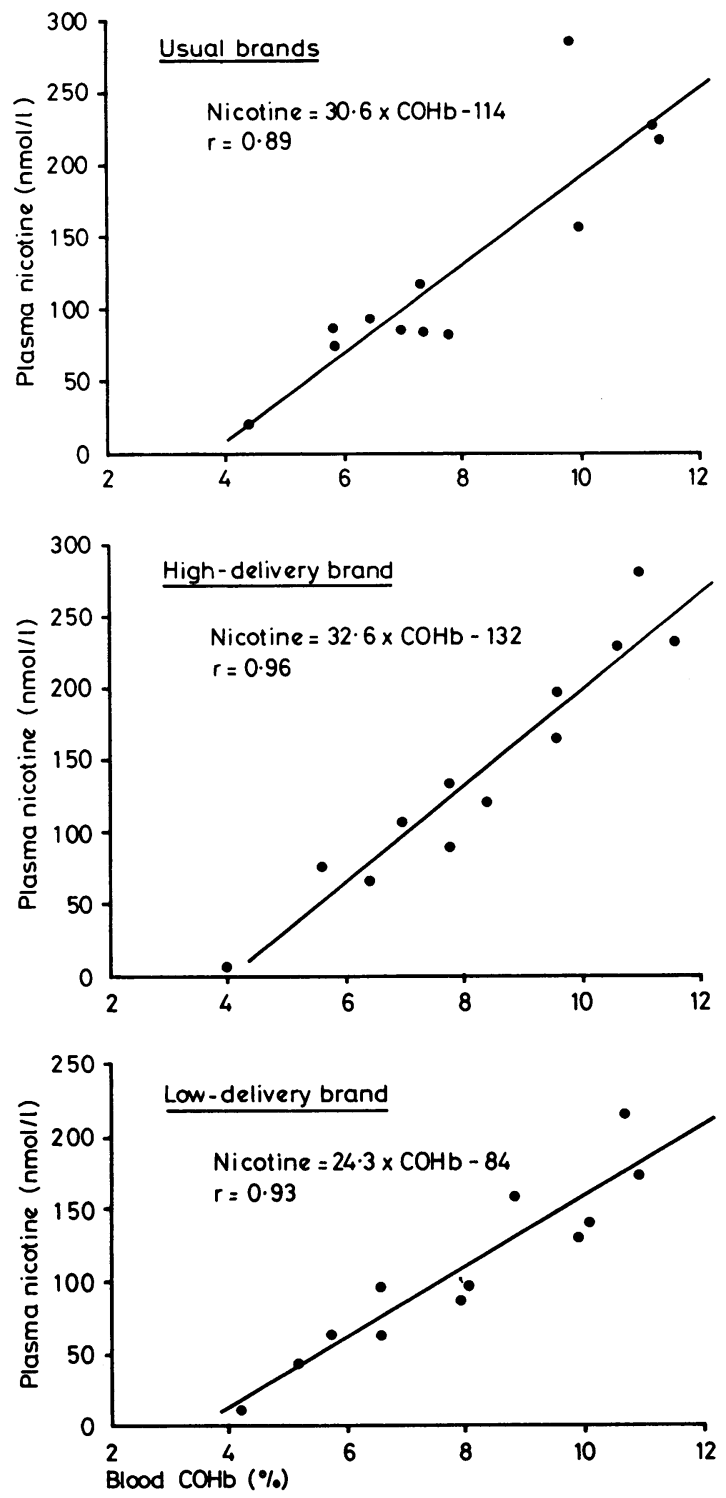

FIG 1-Relation between presmoking plasma nicotine and blood COHb concentrations in 12 smokers for the three brands of cigarette smoked.

Conversion: SI to traditional units-Nicotine: $1 \mathrm{nmol} / \mathrm{l}$ $\simeq 16.2 \mathrm{ng} / 100 \mathrm{ml}$.

brand. Indices of smoking intensity based on the rise in nicotine concentrations during smoking and corresponding values for $\mathrm{COHb}$ were calculated similarly.

The plasma nicotine index suggests that the high-delivery brand was smoked less intensively and the low-delivery brand more intensively than the subjects' usual cigarettes. More importantly, however, the degree of oversmoking or undersmoking indicated by the ratio of observed to expected $\mathrm{COHb}$ concentration agreed closely with that indicated by the ratio of plasma nicotine concentrations. The agreement between the two measures of smoking intensity was close when both presmoking values and the rise with smoking were considered.

In the course of the experiment several variables relating to smoking behaviour, the intake of smoke constituents, and the physiological effects of smoking were measured. Certain of these variables (such as 
weekly cigarette consumption and 24-hour urine nicotine and cotinine excretions) related to cigarettes smoked outside the laboratory; others, such as the rise in heart rate and fall in fingertip temperature, related primarily to a single cigarette smoked in the laboratory. Variables relating to cigarettes smoked outside the laboratory were correlated with presmoking plasma nicotine concentrations, and those relating to the cigarette smoked in the laboratory were correlated with the rise in plasma nicotine concentrations with smoking, to determine whether the degree of association would be large enough to justify using them as a measure of exposure to nicotine. Table $\mathrm{V}$ shows the correlations obtained.

TABLE V-Correlation between plasma nicotine concentrations and other possible measures of nicotine intake (all brands) in 12 smokers

\begin{tabular}{|c|c|c|}
\hline & $\begin{array}{l}\text { Correlation with } \\
\text { plasma nicotine }\end{array}$ & Significance of $r(d f=10)$ \\
\hline \multicolumn{3}{|c|}{ Presmoking concentration } \\
\hline $\begin{array}{l}\text { Presmoking heart rate } \\
\text { Presmoking fingertip temperature } \\
\text { Weekly cigarette consumption } \\
\text { 24-hour cigarette consumption } \\
\text { Mean butt length } \\
\text { 24-hour mouth nicotine intake }\end{array}$ & $\begin{array}{r}-0.09 \\
-0.49 \\
0.60 \\
0.54 \\
-0.73\end{array}$ & $\begin{array}{l}\text { NS } \\
\text { NS } \\
\mathrm{p}<0.05 \\
\mathrm{NS} \\
\mathrm{p}<0.01\end{array}$ \\
\hline $\begin{array}{l}\text { (estimated by butt analysis): } \\
\text { Average intake/cigarette } \\
\text { Total intake }\end{array}$ & $\begin{array}{r}-0.04 \\
0.62\end{array}$ & $\underset{p<0.05}{\text { NS }}$ \\
\hline $\begin{array}{l}\text { 24-hour excretion in urine: } \\
\text { Nicotine* } \\
\text { Cotinine }\end{array}$ & $\begin{array}{l}0.66 \\
0.64\end{array}$ & $\begin{array}{l}\mathrm{p}<0.05 \\
\mathrm{p}<0.05\end{array}$ \\
\hline \multicolumn{3}{|c|}{ Rise in concentration with smoking } \\
\hline $\begin{array}{l}\text { Rise in heart rate } \\
\text { Fall in fingertip temperature }\end{array}$ & $\begin{array}{r}-0.12 \\
0.07\end{array}$ & $\begin{array}{l}\text { NS } \\
\text { NS }\end{array}$ \\
\hline $\begin{array}{l}\text { Laboratory-smoked cigarette: } \\
\text { Butt length } \\
\text { Mouth-level nicotine intake } \\
\text { Proportion of time spent puffing }\end{array}$ & $\begin{array}{r}-0.17 \\
0.17 \\
0.23\end{array}$ & $\begin{array}{l}\text { NS } \\
\text { NS } \\
\text { NS }\end{array}$ \\
\hline
\end{tabular}

$* n=11, d f=9$.

Heart rate and fingertip temperature were not significantly correlated with either resting plasma nicotine concentration or the rise in concentration with smoking. Weekly consumption of cigarettes, however, correlated $(r=0.6)$ with presmoking plasma nicotine concentration and the average length of unsmoked cigarette $(r=-0.73)$. High cigarette consumption and a short butt length would therefore seem to be of some value in predicting greater exposure to nicotine. The total 24-hour mouth-level delivery of nicotine (estimated by analysis of smoked filter tips) was also related in the expected direction to higher plasma nicotine concentrations, although the estimate of nicotine delivery based on the butt from the single laboratory-smoked cigarette was not. Excretions of nicotine and cotinine in a 24-hour collection of urine correlated reasonably well with resting plasma concentrations, and the degree of association was marginally improved $(r=0.7)$ when the amounts of nicotine and cotinine excreted were added together. In general, however, no measure approached that of presmoking $\mathrm{COHb}$ concentration in terms of the strength of its association with plasma nicotine concentrations.

\section{Discussion}

Our data suggest that although the intake of nicotine from a single laboratory-smoked cigarette cannot be estimated on the basis of carbon monoxide intake, a close association nevertheless exists between the longer-term exposure to nicotine and carbon monoxide that is reflected in presmoking blood concentrations. Many epidemiological investigations use a single measurement of $\mathrm{COHb}$ concentration as an indicator of longer-term exposure to nicotine, and we therefore regard as encouraging the close relation found in this study between presmoking plasma nicotine and $\mathrm{COHb}$ concentrations. Once the relation between plasma nicotine and $\mathrm{COHb}$ concentrations has been established for a particular type of cigarette, knowledge of an individual subject's $\mathrm{COHb}$ concentration would enable his plasma nicotine concentration to be estimated with considerable accuracy. Moreover, the close relation between the index of smoking intensity based on $\mathrm{COHb}$ concentration and that based on plasma nicotine concentrations suggests that a measure of carbon monoxide intake alone would serve to indicate the relative exposure to nicotine in populations smoking different types of tobacco product. Our correlations, however, were based on an average of two values for each subject, which may have reduced the variability of the data and therefore increased the degree of association found. The results of a previous study, in which the response of smokers to the dilution of tobacco smoke by ventilated cigarette holders was investigated, were similar; where consistent evidence of compensation for reduced delivery was found the estimate of compensation based on $\mathrm{COHb}$ concentration agreed closely with that based on plasma nicotine concentrations. ${ }^{6}$

The relation between own-brand postsmoking nicotine and $\mathrm{COHb}$ concentrations in the present experiment may be compared with that reported by Russell et $\mathrm{al}^{7}$ for a similar population smoking middle-tar cigarettes (fig 2). Conditions in their experi-

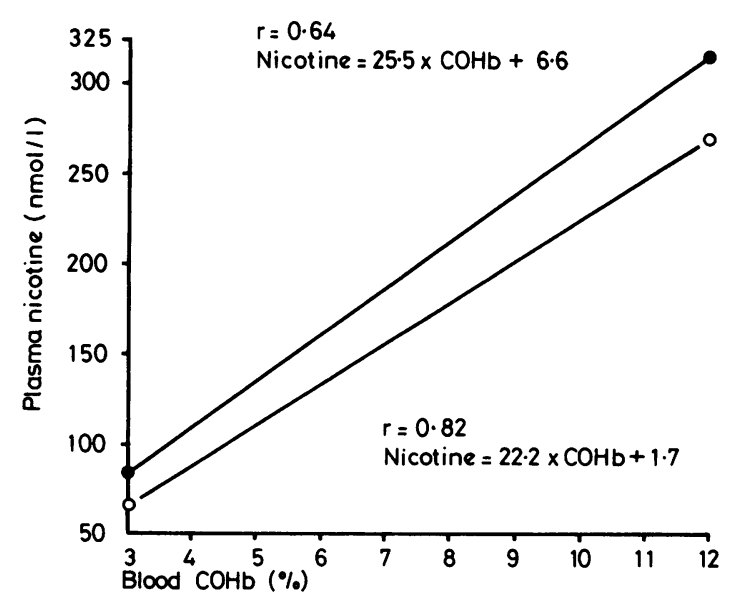

FIG 2-Relation between postsmoking plasma nicotine and blood $\mathrm{COHb}$ concentrations when smokers smoked their usual brands. - - $=$ Relation obtained in this study. $\mathrm{O}-\mathrm{O}=$ Relation obtained in study of Russell et al. ${ }^{7}$

Conversion: SI to traditional units-Nicotine: $1 \mathrm{nmol} / \mathrm{l}$ $\approx 16.2 \mathrm{ng} / 100 \mathrm{ml}$.

ment were slightly different since blood samples were taken midmorning, three minutes after smoking. Nevertheless, the regression of plasma nicotine concentration on $\mathrm{COHb}$ produced a result closely similar to ours though with a slightly higher correlation coefficient, which was probably because each of their 10 subjects was represented four times in the correlation (since blood concentrations were measured on four occasions).

In the present study the correlation between nicotine and $\mathrm{COHb}$ concentrations was higher in the presmoking than postsmoking samples. The explanation for this difference probably lies at least in part in the different pharmacokinetics of the two substances.

We could not follow up experimental evidence of an association between intakes of carbon monoxide and tar because, so far as we know, no measure of tar intake in man is available. Since nicotine and tar are closely allied in the particulate phase of tobacco smoke, exposure to tar might be expected to proceed pari passu with exposure to nicotine. Intake of tar, however, is by deposition and intake of nicotine by absorption, and the proportions of these substances retained by the smoker after inhalation are probably different. The high correlation between exposures to nicotine and carbon monoxide would therefore not in itself justify the conclusion that exposures to carbon monoxide and tar are equally closely associated.

We are grateful to the Tobacco Research Council for funding the research on which this paper is based, Kevin Gough for his advice on statistics, Mr V R Marsh for considerable technical help, and Drs M A H Russell and C Feyerabend for arranging and carrying out the plasma nicotine assays.

Requests for reprints should be sent to Mr R Stepney, Department of Medicine, University of Cambridge Clinical School, Addenbrooke's Hospital, Hills Road, Cambridge CB2 2QQ. 


\section{References}

1 Wald NJ, Idle $M$, Boreham J. Inhaling habits among smokers of different types of cigarette. Thorax (in press).

2 Wald NJ, Idle M, Boreham J. Carbon monoxide in breath in relation to smoking and carboxyhaemoglobin levels. Thorax (in press).

3 Cumming G, Guyatt AR, Holmes MA. The absorption of carbon monoxide from the conducting airways of the human lung. In: Thornton RE, ed. Smoking behaviour: physiological and psychological influences. Edinburgh: Churchill Livingstone, 1978:168-70.

Sjostrand T. The in vitro formation of CO in blood. Acta Physiol Scand $1951 ; 24: 314-32$.
5 Ashton H, Stepney R, Thompson JW. Self-titration by cigarette smokers. Br Med F 1979;ii:357-60.

- Sutton SR, Feyerabend C, Cole PV, Russell MAH. Adjustment of smokers to dilution of tobacco smoke by ventilated cigarette holders. Clin Pharmacol Ther 1978;24:395-405.

7 Russell MAH, Wilson C, Patel UA, Feyerabend C, Cole PV. Plasma nicotine levels after smoking cigarettes with high, medium, and low nicotine yields. $\operatorname{Br} \operatorname{Med} \mathcal{F} 1975 ; \mathrm{ii}: 414-6$.

(Accepted 29 October 1980)

\title{
Feasibility of outpatient management after intra-articular yttrium-90 : comparison of two regimens
}

\author{
P L WILLIAMS, J C W CRAWLEY, A M FREEMAN, D C LLOYD, J M GUMPEL
}

\begin{abstract}
In a study comparing two regimens of treatment after intra-articular irradiation of the knee with yttrium-90 one group of patients was allocated to bed rest for 48 hours in hospital and the other to mobilisation at home. Initially a Robert-Jones orthopaedic bandage was applied to the knee in all patients, serving as a semi-rigid splint, but as loss of isotope from the knee was appreciable in the mobilised patients, subsequent patients were sent home with the knee in a plaster-of-Paris cylinder.

No difference in extra-articular spread or chromosomal damage was found between the patients sent home with their knee in a rigid splint and those treated by bed rest. Clinical outcome at three months was satisfactory in all three groups.

These results show that rigid splinting is essential in reducing extra-articular spread of the isotope but that bed rest is not necessary. Increases in intra-articular pressure associated with quadriceps muscle activity combined with flexion of the knee may be the most important factor affecting extra-articular spread of isotope.
\end{abstract}

\section{Introduction}

Immobilising the knee after intra-articular injection of radioisotope reduces extra-articular spread of isotope, in particular to the regional lymph nodes, ${ }^{12}$ and decreases the incidence of chromosomal damage in peripheral blood lymphocytes. ${ }^{13-5}$ In practice this has necessitated admitting all patients to hospital for bed rest for at least three days after injection. In a recent study, ${ }^{6}$ however, no difference in leakage of isotope or chromosomal damage was found between inpatients confined to bed and those fully mobile in hospital with a rigid splint applied to the treated knee. We carried out the present study to ascertain

\footnotetext{
Northwick Park Hospital and Clinical Research Centre, Harrow, Middlesex HA1 3UJ

P L WILLIAMS, MB, MRCP, medical registrar

J C W CRAWLEY, CENG, MIEE, MRC scientist

A M FREEMAN, MB, DPHYSMED, research assistant

J M GUMPEL, FRCP, consultant physician

National Radiological Protection Board, Harwell, Didcot, Oxfordshire

D C LLOYD, PHD, head, cytogenetics group
}

whether mobilisation at home was acceptable and compared three regimens-namely, bed rest in hospital, mobilisation at home with a Robert-Jones orthopaedic bandage applied to the knee, and mobilisation at home with a plaster-of-Paris cylinder applied to the knee.

\section{Patients and methods}

Twenty-nine intra-articular injections of yttrium-90 silicate were performed on 27 patients with persistent synovitis of the knee (27 single injections, two repeat injections). The synovitis was part of classical or definite rheumatoid arthritis in 14 patients, osteoarthritis in two, and psoriatic arthritis in one; in nine patients the synovitis was confined to one or both knees, while one patient had pigmented villonodular synovitis. The injection dose in each case was $5 \mathrm{mCi}$, except in the patient with pigmented villonodular synovitis, who received $8 \mathrm{mCi}$.

Initially, suitable patients were allocated at random to one of two treatment groups. In both groups a Robert-Jones orthopaedic bandage was applied to the knee immediately after injection, but one group remained in bed in hospital for $\mathbf{4 8}$ hours while the other was allocated to mobilisation at home for $\mathbf{4 8}$ hours, the bandage serving as a semirigid splint. All patients were admitted for assessment the day before injection; the mobilised group went home by car or minicab within two hours after injection. Loss of isotope from the knees was greater in the mobilised group, so that subsequently all suitable patients were sent home with the knee in a plaster-of-Paris cylinder, which served as a rigid splint. The plaster was applied 24 hours before injection, from high on the thigh to just above the ankle, with an aperture to permit access to the knee. The results in those patients were compared with the results in the patients treated with bed rest.

A quantitative hybrid whole-body scanner ${ }^{2}$ was used to detect leakage of isotope from the knee immediately and 48 hours after injection. The retention of isotope in the knee at 48 hours was expressed as a percentage of that present immediately after injection.

Cultures of peripheral blood lymphocytes were prepared by a routine procedure ${ }^{8}$ and analysed for chromosomal damage in patients who had not received previous irradiation. Samples were taken before and at least six weeks after injection and 200 cells scored each time.

Synovitis of both knees and generalised disease activity were assessed clinically immediately before treatment and three, six, and 12 months later. Knee circumference $1 \mathrm{~cm}$ above the patella and minimum heel-buttock distance were recorded, and estimates made of the degree of synovial proliferation, effusion, lateral and anteroposterior stability, extension loss, and valgus or varus deformity. Patients were asked to record their symptoms on each visit. General disease activity was assessed by recording the presence or absence of synovitis in other joints and by measuring grip strength and walking time.

All the patients in the mobilised group were given a questionnaire on which to record any difficulties encountered during the two days spent at home and their preferences about treatment. 